\title{
Anisotropy search in the Ultra High Energy Cosmic Ray Spectrum in the Northern Hemisphere using the Telescope Array surface detector
}

\author{
T. Nonaka ${ }^{* a}$, R. Cady ${ }^{b}$, M. Fukushima ${ }^{a, c}$, D. Ivanov $^{b}$, K. Kawata ${ }^{a}$, E. Kido ${ }^{a}$, \\ J.N. Matthews ${ }^{b}$, S. Nagataki ${ }^{d}$, T. Okuda ${ }^{e}$, H. Sagawa ${ }^{a}$, N. Sakurai ${ }^{f}$, B.T. Stokes ${ }^{b}$, \\ M. Takeda ${ }^{a}$ and A. Taketa ${ }^{g}$, for The Telescope Array Collaboration \\ ${ }^{a}$ Institute for Cosmic Ray Research, University of Tokyo, Kashiwa, Chiba, Japan \\ ${ }^{b}$ High Energy Astrophysics Institute and Department of Physics and Astronomy, University of \\ Utah, Salt Lake City, Utah, USA \\ ${ }^{c}$ Kavli Institute for the Physics and Mathematics of the Universe (WPI), Todai Institutes for \\ Advanced Study, the University of Tokyo, Kashiwa, Chiba, Japan \\ ${ }^{d}$ Astrophysical Big Bang Laboratory, RIKEN, Wako, Saitama, Japan \\ ${ }^{e}$ Department of Physical Sciences, Ritsumeikan University, Kusatsu, Shiga, Japan \\ ${ }^{f}$ Graduate School of Science, Osaka City University, Osaka, Osaka, Japan \\ ${ }^{g}$ Earthquake Research Institute, University of Tokyo, Bunkyo-ku, Tokyo, Japan \\ E-mail: nonaka@icrr.u-tokyo.ac.jp
}

\begin{abstract}
The Telescope Array (TA) experiment is located in the western desert of Utah, USA, and observes ultra high energy cosmic rays in the northern hemisphere. In the region of highest energies, the shape of cosmic ray energy spectrum may contain information on the source density distribution. In this study, we search for directional differences in the shape of energy spectrum. Observed cosmic ray energy spectra are compared between sky areas that have larger density of nearby objects, such as the super-galactic plane, and others that do not.
\end{abstract}

The 34th International Cosmic Ray Conference,

30 July- 6 August, 2015

The Hague, The Netherlands

\footnotetext{
* Speaker.
} 


\section{Introduction}

Ultra high energy cosmic ray (UHECR) primaries lose notable fraction of energy in interactions with photons of cosmic microwave background radiation (CMBR) while propagating distances comparable to the local cosmological structure. The attenuation length depends on a particle type and energy. Protons which have energy larger than $10^{19.7} \mathrm{eV}$ will lose major part of energy in pion photoproduction. Consequently, the spectrum of protons is expected to show suppression of the flux at these energies, which is known as the GZK cut off $[1,2]$. Another relevant process for protons propagating in CMBR is $e^{+}+e^{-}$pair creation. This reaction is important for protons with energies around $10^{18.6} \mathrm{eV}$. As a result, UHECR energy spectra may be different in different areas in celestial sphere. In this work we compare spectra in regions which contain large number of nearby objects with those in a local voids. This approach is complementary to the usual anisotropy studies.

\section{Experiment and analysis}

Telescope Array(TA) experiment [3] employes hybrid approach at energies E $>10^{18} \mathrm{eV}$, and cosmic rays are observed using fluorecence telescopes and surface detector. The surface detector consists of 507 scintillation counters deployed on a square grid with $1.2 \mathrm{~km}$ spacing, covering an area of $670 \mathrm{~km}^{2}$ [4]. The operation of the surface detector started in 2008. The duty cycle of the observation is $95 \%$ on average. Now the exposure is the largest in the northern hemisphere. In this analysis, cosmic-ray events with energies greater than $10^{19} \mathrm{eV}$, collected in a period from May 2008 to May 2013, are used to search for anisotropy related signal in cosmic-ray energy spectrum. From Monte Carlo simulation, the trigger efficiency of cosmic-ray showers at zenith angles of less than $55^{\circ}$ reaches $100 \%$ in the energy range greater than $10^{19} \mathrm{eV}$. Corresponding estimated energy resolution is about $20 \%$, and the angular resolution is $2^{\circ}[3,5]$. The distribution of zenith angles of the observed showers is shown in Fig. 1. In this analysis we divide the sky into two parts. One is the area that contains larger number of nearby objects, whereas the other is the area that contains less nearby objects. The former is called the "On source" area, and the latter is called the "Off source" area. We repeat analyses for two types of the On and Off source areas described below.

\subsection{Analysis for Super Galactic Plane (SGP)}

The Super Galactic Plane (SGP) is a plane which contains more nearby galaxies of our Local Group [6]. The Exposure in TA experiment is almost equally divided when we define a sky within $\pm 30^{\circ}$ of SGP as the On source area, and the other as the Off source area. Fractions corresponding to the On and Off source areas in the total exposure are 52\% and $48 \%$ respectively. The distributions of zenith angles of exposure for the On and Off source areas are plotted in Fig. 2. First we compare the energy distributions of observed air shower events from this On and Off source areas. Fig. 3 shows the energy distributions of the observed showers obtained for the entire exposure, and for On and Off source areas. The shape of the distributions were evaluated by maximum likelihood fit with broken power law. The black line in Fig. 3 shows the best fit broken power law expressed by Eq. 2.1. Here $E_{o}=1 \mathrm{EeV}, C_{0}$ represents the normalization constant proportional to the total number 
of events, while $\alpha_{1,2}$ represent spectrum indexes below $E_{b}$ and above $E_{b}$ respectively.

$$
\begin{gathered}
\frac{\Delta N(E)}{\Delta \log _{10}\left(\frac{E}{E_{o}}\right)}=C_{0}\left(\varepsilon\left(E, E_{b}\right)\left(\frac{E}{E_{o}}\right)^{-\alpha_{1}}+\left(1-\varepsilon\left(E, E_{b}\right)\right)\left(\frac{E}{E_{o}}\right)^{-\alpha_{2}}\right) \\
\varepsilon\left(E, E_{b}\right)=\left\{1:\left(E<E_{b}\right), 0:\left(E>E_{b}\right)\right.
\end{gathered}
$$

The best fit parameters for the energy distribution obtained from the entire exposure are $C_{o}=$ $2.141_{-0.298}^{+0.343} \times 10^{+4}, \alpha_{1}=-1.775_{-0.053}^{+0.053}, \log _{10}\left(E_{b} / E e V\right)=1.778_{-0.068}^{+0.040}$ and $\alpha_{2}=-3.910_{-0.660}^{+0.643}$.

When we evaluate possible difference in energy distributions in On and Off source regions, $\alpha_{1}$ is set to the value obtained from the fit to the distribution for the entire exposure, $C_{0}$ is scaled to the corresponding fraction of the exposure, while $\log _{10}\left(E_{b} / E o\right)$ and $\alpha_{2}$ are set free and obtained from the fitting in corresponding areas. Obtained broken power law functions are plotted in Fig. 3 as solid and dashed lines. Errors for the resulting parameters are shown in Fig. 3 by red and blue contours. Table. 1 summarizes best fit parameters and errors. There are difference in break energy between On source and Off source area. The difference, $\Delta \log _{10}\left(E_{b} / E_{o}\right)$ is 0.16 . For the events fraction in the Off source area above the break energy, $\left(N_{o f f}\left(E>E_{b}\right) / N_{\text {all }}\left(E>E_{b}\right)\right)$, we obtain 0.34 instead of 0.48 which is expected from the exposure ratio. The chance probability was estimated in a simulation which assumes that both distributions are statistically equivalent with the entire exposure. Namely, in each energy bin the events has been shuffled to On and Off source distributions accordingly to the corresponding fraction of the exposure, binomially. At each trial, we obtain a random distribution coming from the same population, and did same evaluation for the distribution difference. Fig. 5 shows the frequency distribution. The horizontal axis corresponds to "Off source" "s break energy and vertical axis corresponds to event fraction above the break energy. Table. 2 shows chance probability to obtain each case. The observed value correspond to a probability $\sim 0.62 \times 10^{-4}(3.2 \sigma)$.

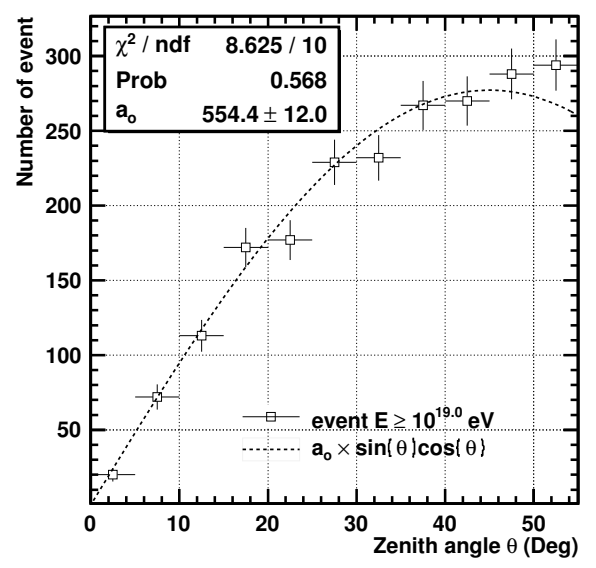

Figure 1: The zenith angle distribution of observed shower events with energy $\mathrm{E} \geq 10 \mathrm{EeV}$.

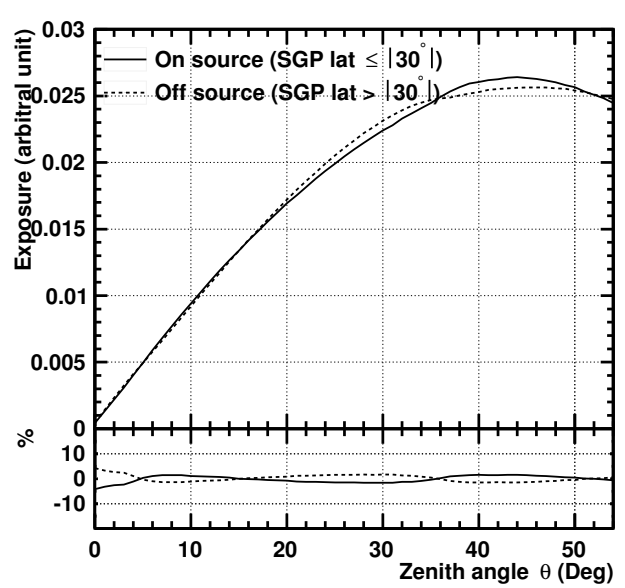

Figure 2: The zenith angle distributions in On and Off source areas. 


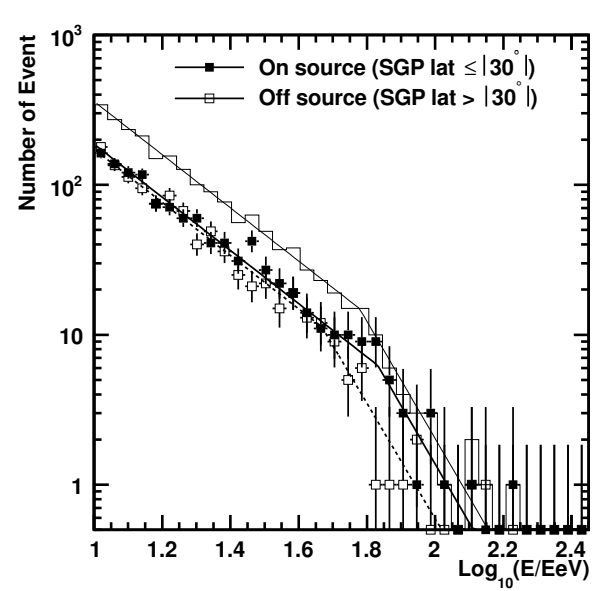

Figure 3: The energy distributions of observed shower events for the On/Off areas using SGP. The black histogram shows distribution of all events. Closed and open symbols show energy distributions observed in On and Off source regions respectively.

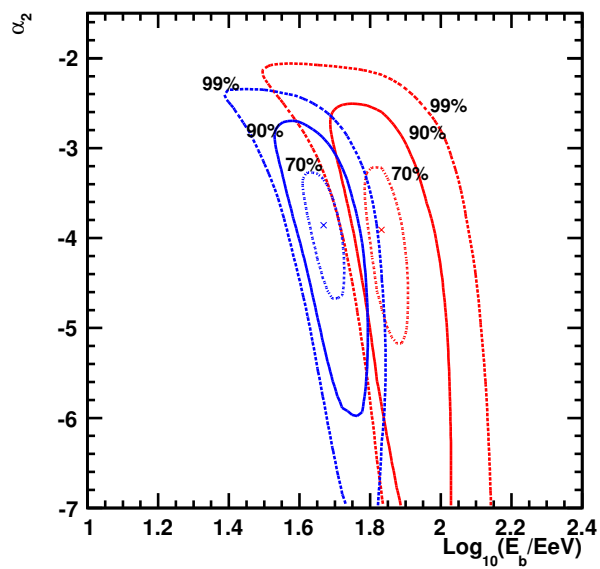

Figure 4: Contours of $\delta \log L$ in the plane of $E_{b}$ and $\alpha_{2}$ in the SGP case. Blue and red colors denote $70 \%, 90 \%$ and $99 \%$ confidence levels for the Off and On source regions respectively.

\begin{tabular}{crrlr}
\hline \hline Region & $C_{o}$ & $\alpha_{1}$ & $\log _{10}\left(E_{b} / \mathrm{EeV}\right)$ & $\alpha_{2}$ \\
\hline All & $2.141_{-0.298}^{+0.343} \times 10^{+4}$ & $-1.775_{-0.053}^{+0.053}$ & $1.778_{-0.0068}^{+0.040}$ & $-3.910_{-0.660}^{+0.643}$ \\
On source & $\left(1.1128 \times 10^{+4}\right)$ & $(-1.775)$ & $1.832_{-0.041}^{+0.069}$ & $-3.910_{-1.260}^{+0.696}$ \\
Off source & $\left(1.0286 \times 10^{+4}\right)$ & $(-1.775)$ & $1.668_{-0.053}^{+0.052}$ & $-3.858_{-0.818}^{+0.582}$ \\
\hline
\end{tabular}

Table 1: Parameters of the best fit broken power law in the SGP case.

\subsection{Analysis for the known object list (VCV list)}

The objects used in this analysis are AGNs from Veron-Cetty\& Veron 12 catalogue with cut on redshift $\mathrm{z}<0.018$ [7]. This is same object list which was already used at other correlation studies, see e.g.[8]. The definition of the On and Off source areas was adjusted by changing the size of the opening angle from $1^{\circ}$ to $15^{\circ}$ to maximize the signal. It was done in the following way. For a given opening angle and given energy bin in the Off source area we calculate the ratio of the observed number of events to the expected number, based on the exposure ratio. Then we calculate $\chi^{2}$ deviation of this quantity from the expectation, and sum over all bins. We choose opening angle on the criteria that this sum is largest. The largest deviations occurs for the opening angle $11^{\circ}$ and the result does not depend upon binning. For this opening angle the ratio of the observed number of events to the expected number in the Off source area is shown in Fig. 6. In this case the exposure fractions are 0.81 and 0.19 for the On and Off source areas, respectively. The zenith angle distributions are plotted in Fig. 7. The exposure for the On and Off source area deviate from the geometrical exposure insignificantly. Fig. 8 shows energy distributions for the overall dataset, On source area and Off source area. We fit these distriutions by the same broken power low as in the previous section. The best fit parameters are summarised in Table. 3. Corresponding confidence contours are shown in Fig. 9. Break energies in the On and Off source regions differ by a factor $\Delta \log _{10}\left(E_{b} / E_{o}\right) \sim 0.31$. The event fraction in the Off source region above the break energy 


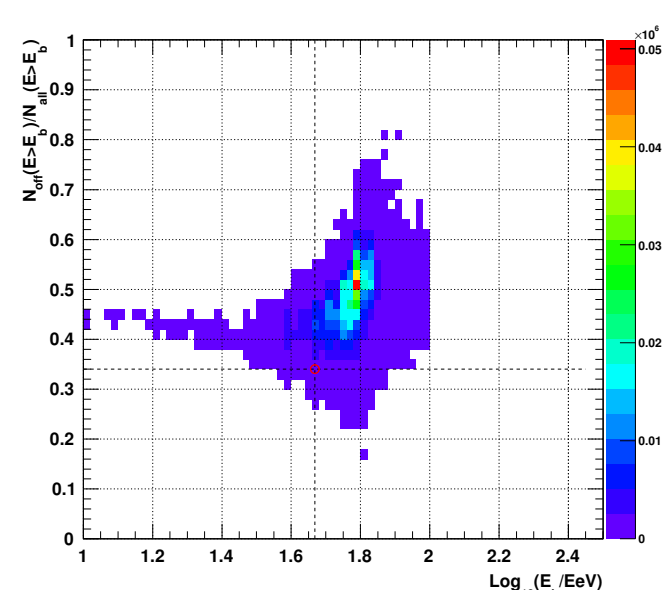

Figure 5: Event fraction in the Off source region above the break energy versus $\log _{10}\left(E_{b} / E e V\right)$ obtained in Monte Carlo simulations for the SGP case.

\begin{tabular}{crc}
\hline \hline Condition & N & Fraction \\
\hline$E_{b}>10^{1.668} \mathrm{EeV}, \frac{N_{\text {off }}\left(E>E_{b}\right)}{N_{\text {all }}\left(E>E_{b}\right.}>0.34$ & 41580 & 0.83177 \\
$E_{b}>10^{1.668} \mathrm{EeV}, \frac{N_{\text {off } f}\left(E>E_{b}\right)}{N_{\text {all }}\left(E>E_{b}\right.}<0.34$ & 7996 & 0.15996 \\
$E_{b}<10^{1.668} \mathrm{EeV}, \frac{N_{\text {off }}\left(E>E_{b}\right)}{N_{\text {all }}\left(E>E_{b}\right.}<0.34$ & 31 & 0.00062 \\
$E_{b}<10^{1.668} \mathrm{EeV}, \frac{N_{\text {off }}\left(E>E_{b}\right)}{N_{\text {all }}\left(E>E_{b}\right.}>0.34$ & 383 & 0.007662 \\
\hline
\end{tabular}

Table 2: The number of occurrences with stated conditions in the SGP case. The estimated chance probability to obtain larger deviation is $6.2 \times 10^{-4}$.
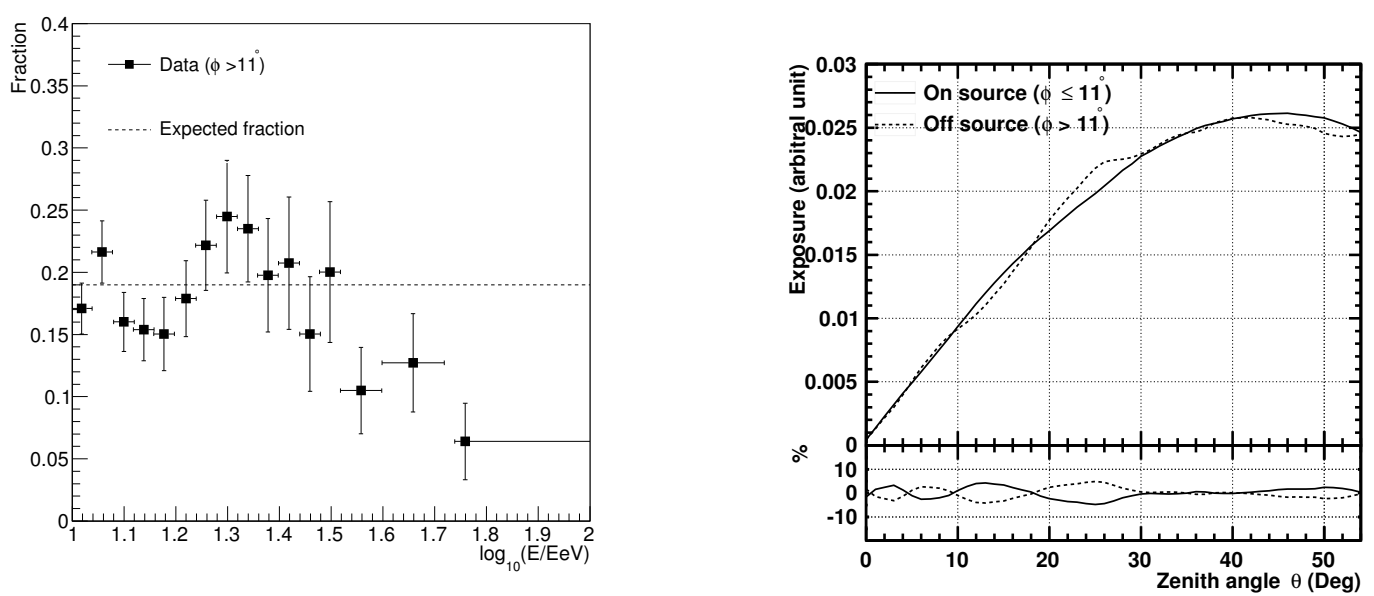

Figure 6: Observed number of events to the expected number in the Off source area as a function of energy. Opening angle corresponds to $11^{\circ}$, while $\chi^{2}$ is largest, $\chi^{2} / n d o f=1.66$

Figure 7: Zenith angle distributions in the On and Off source regions.

$\left(N_{o f f}\left(E>E_{b}\right) / N_{\text {all }}\left(E>E_{b}\right)\right)$ is 0.12 instead of 0.19 which is expected from the exposure ratio. Corresponding chance probability is shown Fig. 10 and Table. 4. It was estimated using the same procedure that was applied for the SGP analysis. A penalty factor which accounts for the opening angle tuning was calculated in the Monte Carlo simulation. The estimated penalty factor is 9 .

\subsection{Systematic error}

In principle, time variation of the energy scale due to change in atmospheric conditions can be the source of systematic error, though it is supposed to be averaged for over 5 years of observations. Anyway, to check its influence, the event rate with energies greater than $10^{19.0} \mathrm{eV}$ was studied in 


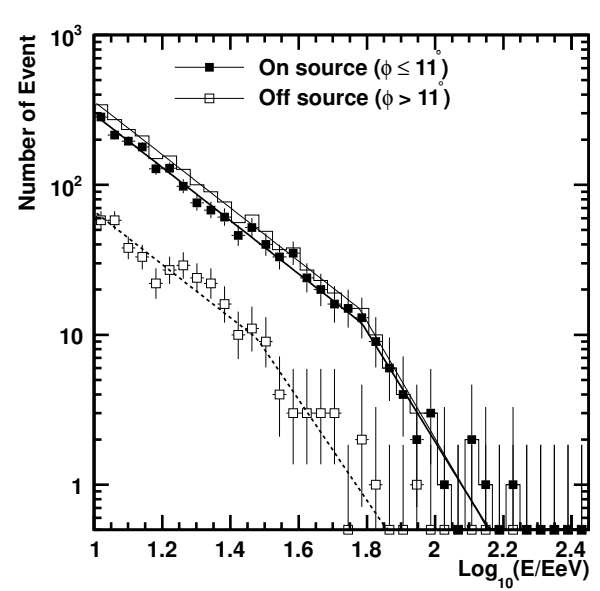

Figure 8: The energy distributions of observed shower events for the On/Off areas using AGN. The black histogram shows distribution of all events. Closed and open symbols show energy distributions observed in On and Off source regions respectively.

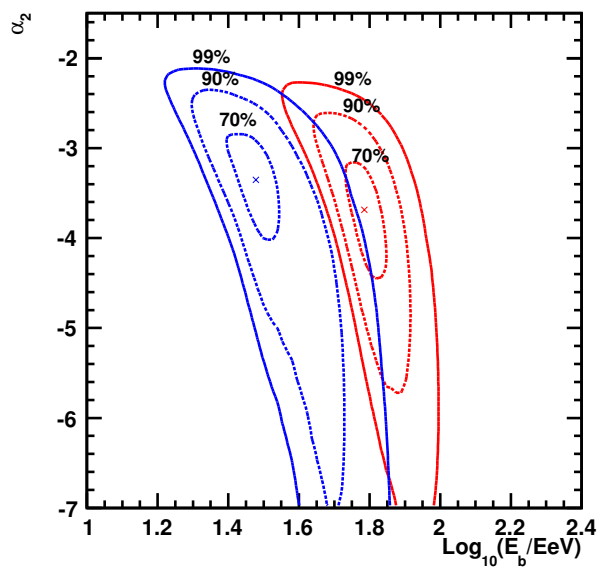

Figure 9: Contours of $\delta \log L$ in the plane of $E_{b}$ and $\alpha_{2}$ in the AGN case. Blue and red colors denote $70 \%$, $90 \%$ and $99 \%$ confidence levels for the Off and On source regions respectively.

\begin{tabular}{crrlr}
\hline \hline Region & $C_{o}$ & $\alpha_{1}$ & $\log _{10}\left(E_{b} / \mathrm{EeV}\right)$ & $\alpha_{2}$ \\
\hline All & $2.141_{-0.298}^{+0.343} \times 10^{+4}$ & $-1.775_{-0.053}^{+0.053}$ & $1.778_{-0.068}^{+0.040}$ & $-3.910_{-0.660}^{+0.643}$ \\
On source & $\left(1.7336 \times 10^{+4}\right)$ & $(-1.775)$ & $1.786_{-0.046}^{+0.058}$ & $-3.663_{-0.784}^{+0.515}$ \\
Off source & $\left(4.0782 \times 10^{+4}\right)$ & $(-1.775)$ & $1.470_{-0.084}^{+0.061}$ & $-3.352_{-0.673}^{+0.529}$ \\
\hline
\end{tabular}

Table 3: Parameters of the best fit broken power law in the AGN case.

anti-sidereal time [9]. The fluctuation amplitude of the event rate in the time bin is at most 5\% $\pm 3 \%$. Given observed spectral index at around $10^{19.0} \mathrm{eV}$, this corresponds to the energy shift by $2.5 \%$. After considering this shift, the chance probability for the observed difference of energy distributions in SGP analysis is still $6.9 \times 10^{-4}(3.2 \sigma)$.

\section{Summary and discussion}

In this analysis, a new approach to search for the anisotropy of UHECR is developed. It employs modulation of the energy spectrum due to an energy losses in CMBR during propagation of primaries. The energy distributions of observed events were fitted by a broken power low. Energy distributions within $30^{\circ}$ from SGP and out of this region were compared. Results are summarised in Table. 1. These distributions differ. Chance probability to obtain such difference in statistically equivalent distributions is estimated as $\sim 6.2 \times 10^{-4}(3.2 \sigma)$. Similarly, observed energy distributions of events within $11^{\circ}$ from VCV AGNs and out of this region were compared. The result is summarised in Table. 3. Again, distributions differ. Chance probability to obtain such difference in statistically equivalent distributions is estimated as $\sim 1.5 \times 10^{-2}(2.2 \sigma)$ after considering penalty factor for the scan in opening angle. In Off source regions the flux has steeper suppression than in On source regions. 


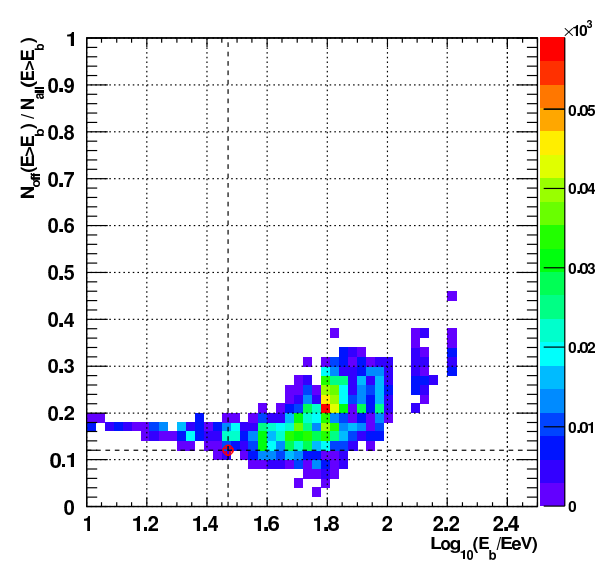

Figure 10: Event fraction in the Off source region above the break energy versus $\log _{10}\left(E_{b} / E e V\right)$ obtained in Monte Carlo simulations for the AGN case.

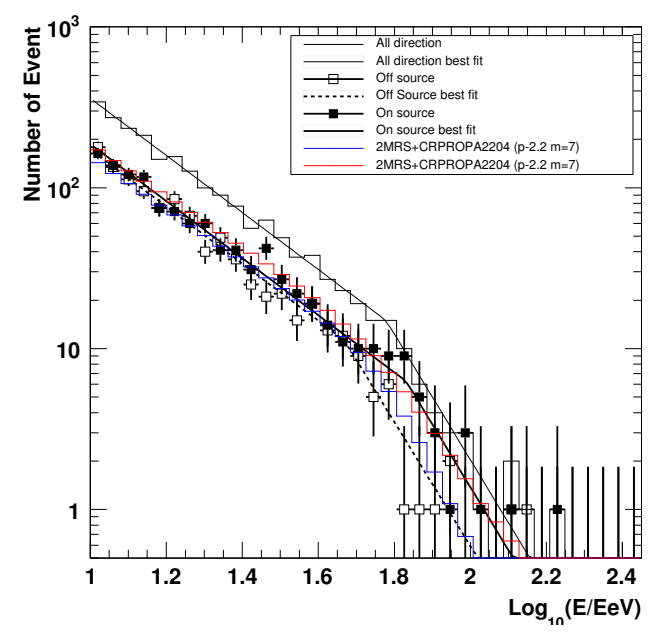

Figure 11: Comparison of energy distributions expected for protons arriving from the sources with injection index of -2.2 , evolution parameter of 7 and 2MRS density profile.

\begin{tabular}{crc}
\hline \hline Condition & N & Fraction \\
\hline$E_{b}>10^{1.470} \mathrm{EeV}, \frac{N_{\text {off }}\left(E>E_{b}\right)}{N_{\text {all }}\left((\Sigma) E_{b}\right.}>0.12$ & 2004 & 0.845 \\
$E_{b}>10^{1.470} \mathrm{EeV}, \frac{N_{\text {off }}\left(E>E_{b}\right)}{N_{\text {all }}\left(E>E_{b}\right.}<0.12$ & 213 & 0.090 \\
$E_{b}<10^{1.470} \mathrm{EeV}, \frac{N_{\text {off }}\left(E>E_{b}\right)}{N_{\text {all }}\left(E>E_{b}\right.}<0.12$ & 4 & $1.7 \times 10^{-3}$ \\
$E_{b}<10^{1.470} \mathrm{EeV}, \frac{N_{\text {off }}\left(E>E_{b}\right)}{N_{\text {all }}\left(E>E_{b}\right.}>0.12$ & 152 & 0.0064 \\
\hline
\end{tabular}

Table 4: The number of occurrences with stated conditions in the AGN case. Chance probability to obtain larger deviation is $\sim 1.5 \times 10^{-2}$ after considering penalty factor for the scan in the opening angle.

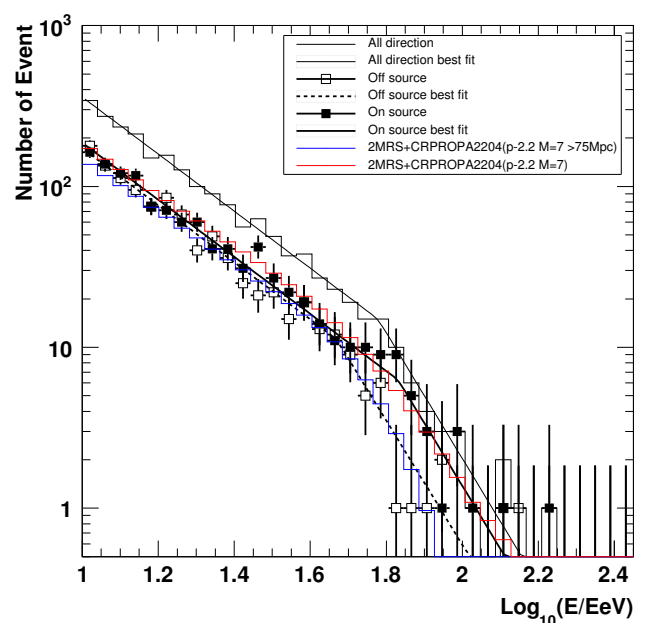

Figure 12: Same figure with Fig. 16 with artificial cut on source distribution at off source side $<75 \mathrm{Mpc}$.

To model observed differences in energy distributions in Monte Carlo, we performed simulations using a propagation code CRPropa2.2.0.4 [10] and the source distribution from the 2MRS catalogue [11] using the density profile calculation described in [12]. Fig. 11 and Fig. 12 display results. Here injection index and evolution parameter were set to -2.2 and 7, respectively [13]. Qualitatively, the difference of observed energy distributions between the On source and Off source regions was reproduced well by this simulation. We conclude that there is strong indication for flux differences of UHECR in different regions of the sky in the Northern hemisphere. We believe that the approach developed here will help to reveal cosmic ray sources and their chemical composition. 


\section{Acknowledgements}

Latest Telescope Array collaborators and acknowledgement is listed in the URL ${ }^{1}$.

\section{References}

[1] K. Greisen, End to the Cosmic-Ray Spectrum?, Physical Review Letters 16 (Apr., 1966) 748-750.

[2] G. T. Zatsepin and V. A. Kuz'min, Upper Limit of the Spectrum of Cosmic Rays, Soviet Journal of Experimental and Theoretical Physics Letters 4 (Aug., 1966) 78.

[3] T. Abu-Zayyad et al., The cosmic-ray energy spectrum observed with the surface detector of the telescope array experiment, The Astrophysical Journal Letters 768 (2013), no. 1 L1.

[4] T. Abu-Zayyad et al., The surface detector array of the telescope array experiment, Nuclear Instruments and Methods in Physics Research Section A: Accelerators, Spectrometers, Detectors and Associated Equipment 689 (2012), no. 087 - 97.

[5] Telescope Array Collaboration, CORSIKA Simulation of the Telescope Array Surface Detector, ArXiv e-prints (Mar., 2014) [arXiv:1403.0644].

[6] G. de Vaucouleurs, Evidence for a local super,galaxy, aj 58 (Feb., 1953) 30.

[7] M.-P. Véron-Cetty and P. Véron, A catalogue of quasars and active nuclei: 12th edition, Astronomy and Astrophysics 455 (Aug., 2006) 773-777.

[8] J. M. Neto and the Pierre Auger Collaboration, Anisotropy studies with the pierre auger observatory, Journal of Physics: Conference Series 409 (2013), no. 1012108.

[9] F. J. M. Farley and J. R. Storey, The sidereal correlation of extensive air showers, Proceedings of the Physical Society. Section A 67 (1954), no. 11996.

[10] K.-H. Kampert, J. Kulbartz, L. Maccione, N. Nierstenhoefer, P. Schiffer, G. Sigl, and A. R. van Vliet, \{CRPropa\} 2.0 a public framework for propagating high energy nuclei, secondary gamma rays and neutrinos, Astroparticle Physics 42 (2013), no. 041 - 51.

[11] J. P. Huchra, L. M. Macri, K. L. Masters, T. H. Jarrett, P. Berlind, M. Calkins, A. C. Crook, R. Cutri, P. Erdodu, E. Falco, T. George, C. M. Hutcheson, O. Lahav, J. Mader, J. D. Mink, N. Martimbeau, S. Schneider, M. Skrutskie, S. Tokarz, and M. Westover, The 2 mass redshift surveyâĂ ̌̌description and data release, The Astrophysical Journal Supplement Series 199 (2012), no. 226.

[12] H. B. J. Koers and P. Tinyakov, Flux calculations in an inhomogeneous universe: weighting a flux-limited galaxy sample, Monthly Notices of the Royal Astronomical Society 399 (2009), no. 2 1005-1011, [http://mnras.oxfordjournals.org/content/399/2/1005.full.pdf+html].

[13] E.Kido et al., Propagation simulations, Proceedings of UHECR2014 conference (2014).

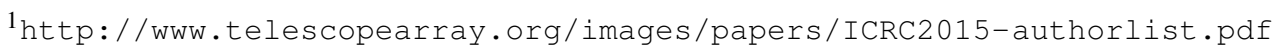

